\title{
lonospheric Time-delay over Akure Using Global Positioning System Observations
}

\author{
Olawale S. BOLAJI ${ }^{1}$, Patrick A. IZANG ${ }^{2,3}$, Olakunle R. OLADOSU ${ }^{2,3}$, \\ Femi KOYA ${ }^{4}$, Rufus S. FAYOSE ${ }^{5,6}$, and Akeem B. RABIU ${ }^{3,6}$ \\ ${ }^{1}$ Department of Physics, University of Lagos, Akoka, Nigeria \\ e-mail: oloriebimpjch2002@yahoo.co.uk \\ ${ }^{2}$ African Regional Centre for Space Science Technology Education (English), \\ Obafemi Awolowo University, Ile Ife, Nigeria \\ ${ }^{3}$ National Space Research and Development Agency (NASRDA), Abuja, Nigeria \\ ${ }^{4}$ Department of Mechanical Engineering, Obafemi Awolowo University, \\ Ile Ife, Nigeria \\ ${ }^{5}$ Department of Physics, Adekunle Ajasin University, Akungba-Akoko, Nigeria \\ ${ }^{6}$ Space Physics Laboratory, Department of Physics, \\ Federal University of Technology, Akure, Nigeria
}

\begin{abstract}
Ionospheric time delay $(V \Delta t)$ variability using Global Positioning System (GPS) data over Akure $\left(7.15^{\circ} \mathrm{N}, 5.12^{\circ} \mathrm{E}\right)$, Nigeria, has been studied. The observed variability of $V \Delta t$ in comparison to older results of vertical total electron content (TEC) across similar regions has shown equivalent signatures. Higher monthly mean values of $V \Delta t(M V \Delta t)$ were observed during daytime as compared to nighttime (pre- and postmidnight) hours in all months. The highest $M V \Delta t$ observed in September during daytime hours range between $\sim 6$ and $\sim 21 \mathrm{~ns}(\sim 1.80$ and $\sim 6.30 \mathrm{~m})$ and at post-midnight, they are in the range of $\sim 1$ to $\sim 6 \mathrm{~ns} \quad(\sim 0.3$ to $\sim 1.80 \mathrm{~m}$ ). The possible mechanisms responsible for this variability were discussed. Seasonal $V \Delta t$ were investigated as well.
\end{abstract}

Keywords: ionosphere, Global Positioning System, time delay, total electron content. 


\section{INTRODUCTION}

Navigation systems have witnessed tremendous improvements in the past four decades. One of such areas of achievements is the invention of Global Positioning System (GPS) by the Department of Defence (DoD), United States of America (USA), in the 1990s. GPS positioning $(P)$, velocity $(V)$, and time $(T)$ accuracy from the GPS receiver records are strongly affected by the ionosphere activities. Our major concern regarding this work is about the GPS radio wave signal propagation within the ionosphere and not the troposphere. Bolaji (2012) restricted the review of the atmospheric propagation on the GPS signal to geometric effects. From the geometric effect, he reported that signal absorption effects are not important because the restriction assumed that all changes in the medium are smaller within one wavelength of signal (in the case of GPS, this is about $20 \mathrm{~cm}$ ). Electromagnetic (EM) wave interactions with the ionosphere are well understood from the previous works of Ginzburg (1970), Chen (1984), Davies (1990), McNamara (1991), Hofmann-Wellenhof et al. (2012), and Odijk (2002). They reported that when EM wave propagates in free space, its velocity is approximately equal to the velocity of light $\left(3.0 \times 10^{8} \mathrm{~m} / \mathrm{s}\right)$, so that the refractive index is equal to 1 . However, when EM wave propagates in the ionosphere, its velocity change; this is due to its interaction with particles present within the ionospheric medium. Therefore, GPS signals traversing through ionospheric medium to the receiving point are expected to propagate along a straight and geometric line, but, in a refractive medium like ionosphere, they bend due to refraction. In this scenario, the wave could be in phase (advance) and the refractive index will be smaller than 1; otherwise, if the wave is in group (delay), the refractive index is larger than 1 . This indicates that, in the ionosphere, the phase of the wave is advancing $(<1)$ and its group is delayed $(>1)$. Ratcliffe (1975) suggested that the ionospheric refractive index is not constant, so that it existed with varying densities of charged particles because it is an inhomogeneous medium. Apart from being an inhomogeneous medium, its anisotropy characteristic explains why the GPS waves are circularly polarized by Faraday rotation phenomenon (Hall et al. 1996).

Besides inhomogeneous and anisotropic phenomena, the ionosphere is a dispersive medium, that is, the phase velocity of a wave is a function of its frequency. All these inhomogeneous, anisotropic, and dispersive phenomena are well-represented in the Appleton-Hatree formula (Eq. 1) when the absorption effects due to collisions between the electrons are ignored.

$$
n_{\varphi, j}^{i p} \pm=\sqrt{1-\frac{X_{j}}{1-\frac{Y_{T, j}^{2}}{2\left(1-X_{j}\right)} \pm \sqrt{\frac{Y_{T, j}^{4}}{4\left(1-X_{j}\right)^{2}}+Y_{L, j}^{2}}}},
$$


where $X_{j}=f_{p}^{2} / f_{j}^{2}, Y_{T, j}$, and $Y_{L, j}$ are the transversal and longitudinal components of $Y_{j} . Y_{j}=f_{g} / f_{j}, f_{j}$ is the GPS signal frequency and $f_{g}$ is the gyro frequency. So, $Y_{T, j}=Y_{j}|\sin \theta|$ and $Y_{L, j}=Y_{j}|\cos \theta|, \theta$ being the angle between $Y_{j}$ and $Y_{L, j}$. This is also applicable to $Y_{j}$ and $Y_{T, j}$. The frequency $f_{p}$ known as the electron plasma frequency is the natural frequency of oscillation for a slab of neutral plasma after the electrons have been displaced from the ions and are able to move freely. This is computed as follows:

where

$$
f_{p}=\sqrt{A N_{e}}
$$

$$
A=\frac{e^{2}}{4 \pi^{2} m_{e} \varepsilon_{0}} \approx 80.6 \mathrm{~m}^{3} / \mathrm{s}^{2} .
$$

$N_{e}$ denotes the free electron density in $\mathrm{m}^{-3}$. The value for $A$ in Eq. 2 was obtained from the natural constants $e=1.60218 \times 10^{-19}$ coulomb for the electron charge, $m_{e}=9.10939 \times 10^{-31} \mathrm{~kg}$ for the electron mass and $\varepsilon_{0}=$ $8.85419 \times 10^{-12} \mathrm{farad} / \mathrm{meter}$ for the permittivity of free space.

The inhomogeneity of the ionosphere is reflected in the free electron density $\left(N_{e}\right)$, which is not a constant, but is a function of place and time. The dispersive nature of the ionosphere is recognized from its dependence on the frequency of the wave. The anisotropic part is a function of $f_{g}$. The doublerefraction is reflected by the \pm sign, which means that either a plus or minus sign can be used, depending on the polarization of the wave. A positive sign corresponds to the left-handed circularly polarized wave (the extraordinary wave) and a negative sign indicates a right-handed circularly polarized wave (the ordinary wave). For GPS estimates, ordinary wave having minus sign is significant.

By comparing the phase and group propagation effects using the ionospheric first order, higher orders and total electron content (TEC), the GPS time delay observation can be made. In summary, the first-order phase effect is equal in magnitude but opposite in sign to the first-order group effect. The second and third order phase effects posses opposite signs compared to their corresponding group effect counterparts, but they do not have the same magnitude. The second order phase effect is half the second order group effect, while the third order phase effect is one third of the third order group effect. The fourth order phase and group effects are the same. From the first order ionospheric delay, which is a function of the integral term $\int N_{e} d p$, it is equivalent to the TEC, which is the number of free electrons in a tube of 1-meter square cross section along the geometric line from the receiver to satellite. The tube of 1-meter square cross section along the geo- 
metric line is a function of the signal bend due to ionospheric refraction, which represents the area (distance covered) where the ionospheric delay occurs. TEC is often expressed in total electron content units (TECU) and $1 \mathrm{TECU}=10^{16}$ electron $/ \mathrm{m}^{2}$. Using this definition of TEC, the first-order delay can be rewritten as:

$$
i_{g, j}^{(1)}=\frac{A}{2 f_{j}^{2}} \text { TEC where } \mathrm{A} \approx 80.6 \mathrm{~m}^{3} / \mathrm{s}^{2} .
$$

Therefore, the first order delay is equivalent to the distance covered $\left(D C_{d}\right)$ around the tube where the ionospheric delay occurs:

$$
i_{g, j}^{(1)}=D C_{d}
$$

and

$$
D C_{d}=\Delta t C .
$$

From Eq. 5, $\Delta t$ is the time delay experienced by the GPS signal when traversing through the ionosphere and $C$ is the speed of light.

Interestingly, the TEC estimates (Eq. 3), which were originally aimed at correcting the ionospheric error due to electron density population from the GPS radio signal when travelling through the ionosphere could provide an extraordinary ionospheric research regarding ionospheric time delay. Therefore, the variability of vertical time delay over the Nigeria ionosphere, its influences on terrestrial applications and possible mechanisms responsible for its variability will be investigated.

\section{MATERIALS AND METHODOLOGY}

Records from Global Positioning System (GPS) receiver located at the Federal University of Technology (FUT), Akure, Nigeria (geographic latitude $7.15^{\circ} \mathrm{N}$, geographic longitude $5.12^{\circ} \mathrm{E}$ ) are employed. Data-set from 5 months (January, March, August, September, and October) during the year 2010 were analyzed. The remaining months are not available because they are associated with larger data gap due to inconsistent power supply. Despite this shortcoming, the available data accommodate all seasons. The Solar Influences Data Analysis Center (SIDC 2013) classified year 2010 as an ascending phase of solar activity with an annual sunspot number of 16.5. This classification results from comparing annual sunspot numbers of previous years 2008 and 2009. The years 2008 and 2009 have an annual sunspot numbers of 2.9 and 3.2, respectively. Estimates of the uncorrected slant total electron content $\left(S_{u}\right.$ TEC) measured at every 1 minute interval from records of GPS receiver at FUT, Akure, Nigeria, were corrected for satellite differential delay $\left(b_{s}\right)$, receiver differential delay $\left(b_{R}\right)$ and the receiver inter-channel bias $\left(b_{R X}\right)$. These corrected $S_{u}$ TEC values were then converted to vertical to- 


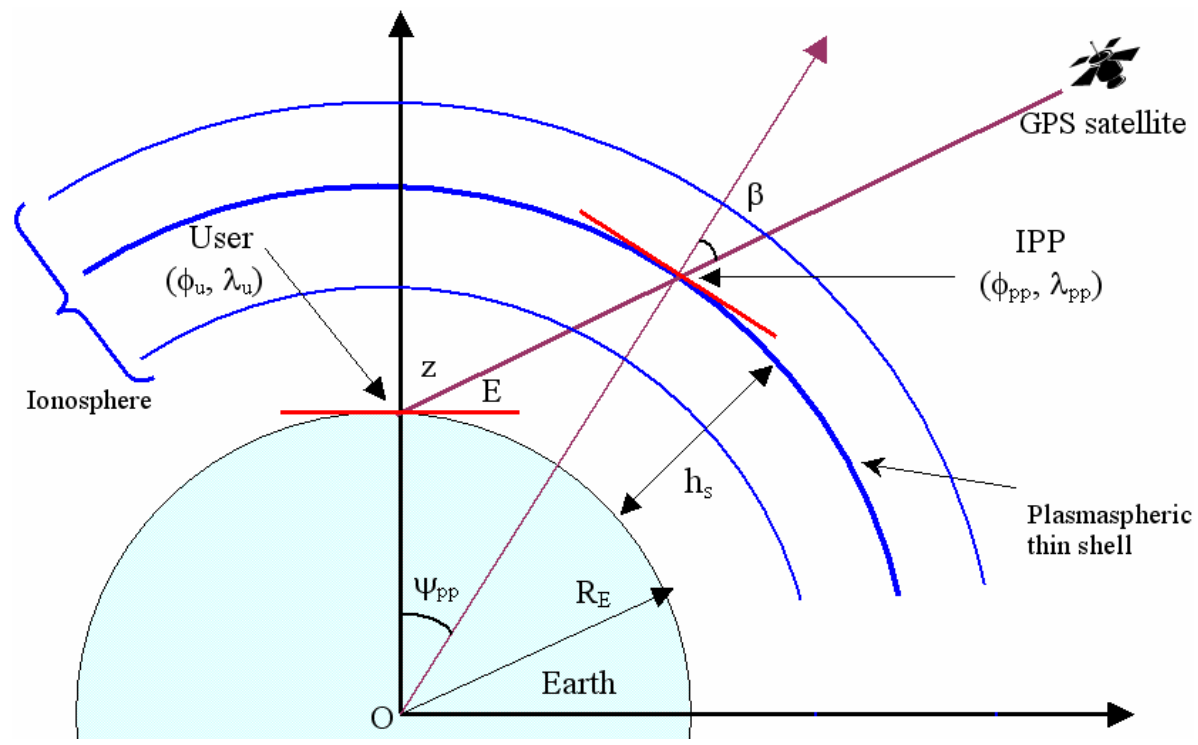

Fig. 1. The vertical total electron content $\left(V_{c}\right.$ TEC) deduced from slant total electron content $\left(S_{u}\right.$ TEC) using thin shell approximation with an ionospheric height $\left(h_{s}\right)$ of $350 \mathrm{~km}$.

tal electron content $\left(V_{c}\right.$ TEC) using thin shell approximation with an ionospheric height of $350 \mathrm{~km}$. Figure 1 shows graphical representation of these analyses and the mathematical expressions for it are as follows:

$$
V_{c} \mathrm{TEC}=S_{u} \mathrm{TEC}-\left[b_{R}+b_{S}+b_{R X}\right] / S(E),
$$

where $S(E)$ is the obliquity factor with zenith angle $(z)$ at the Ionospheric Pierce Point (IPP), $E$ is the elevation angle of the satellites in degrees, and $V_{c}$ TEC is the vertical TEC at the IPP. The $S(E)$ is defined by Mannucci et al. (1993) and Langley et al. (2002) as:

$$
S(E)=\frac{1}{\cos (z)}=\left\{1-\left(\frac{R_{E} \times \cos (E)}{R_{E}+h_{S}}\right)^{2}\right\}^{-0.5},
$$

where $R_{E}$ is the mean radius of the Earth, measured in kilometers [km], and $h_{s}$ is the height of the ionosphere from the surface of the Earth $(\sim 350 \mathrm{~km})$. These analyses from Eqs. 6 and 7 were put together in C-programming language developed by S.G. Krishna (ISR 2009). This software was used to compute $V_{c}$ TEC values for each month under investigation. This same procedure was employed by Bolaji et al. $(2012,2013)$ to compute $V_{c}$ TEC. The time convention for these analyses is in universal time (UT), but, in this pa- 
per, local time (LT) will be used and Nigeria is 1 hour ahead of Greenwich Mean Time (GMT). Therefore, 12:00 UT is 13:00 LT in Nigeria. Now, substituting TEC and $i_{g, j}^{(1)}$ in Eq. 3, with $V_{c}$ TEC in Eq. 6 and $D C_{d}$ with Eq. 4 , in that order, result to

$$
\Delta t=\frac{A}{2 C f_{j}^{2}} V_{c} \mathrm{TEC} .
$$

All meanings of terms from the above equations remained unchanged. The vertical $\Delta t(V \Delta t)$ on each hour of a day over the 5 months were deduced. For each month, mean values of $V \Delta t(\mathrm{M} V \Delta t)$ are estimated. Eleman (1973) classified months according to the Lloyd's seasonal pattern of three seasons: December Solstice (January, February, November, December), Equinoctial Season (March, April, September, October), and June Solstice (May, June, July, August). Since each month under investigation in this study falls within the Lloyd's seasonal pattern, each month will represent each season. That is, March, September, and October will represent Equinox Season. The month of August will represent June Solstice (Summer Season) and December will represent December Solstice (Winter Season). The seasonal variations of $V \Delta t(S S V \Delta t)$ for all the months under investigation are estimated by finding the average of the $\mathrm{M} V \Delta t$ under a particular season.

\section{RESULTS AND DISCUSSIONS}

\subsection{Variability of the ionospheric monthly vertical time delay (MV $V t$ )}

For clearer representation of monthly mean hourly values of $V \Delta t(\mathrm{M} V \Delta t)$ during daytime, pre-, and post-midnight hours, September with highest magnitudes of $\mathrm{M} V \Delta t$ was divided into daytime (07:00-18:00 LT), pre-midnight (19:00-24:00 LT), and post-midnight (00:01-06:00 LT) hours (Fig. 1). In Figure 1, the top panel shows daytime $\mathrm{M} V \Delta t$ variability, the bottom lefthand panel shows pre-midnight $\mathrm{M} V \Delta t$ variability, and the bottom right-hand panel shows post-midnight $\mathrm{M} V \Delta t$ variability. The magnitudes of $\mathrm{M} V \Delta t$ were as well estimated over all hours for January, March, August, and October (Figs. 2-5). Figures 2-6 show September, January, March, August, and October $\mathrm{M} V \Delta t$ variability with bar-charts coloured in blue, in that order. The $\mathrm{M} V \Delta t$ magnitudes measured in nano seconds [ns] are plotted on the $y$ axis, with local time (LT) on the $x$ axis. Figure 7 shows the monthly mean variability of total electron content (TEC) on all hours. The September, January, March, August, and October variability of TEC is in dotted black lines with a downward-pointing triangle, red lines with a square, green lines with a diamond, blue lines with a five-pointed star, and magenta lines with a righthand pointing triangle, in that order. 

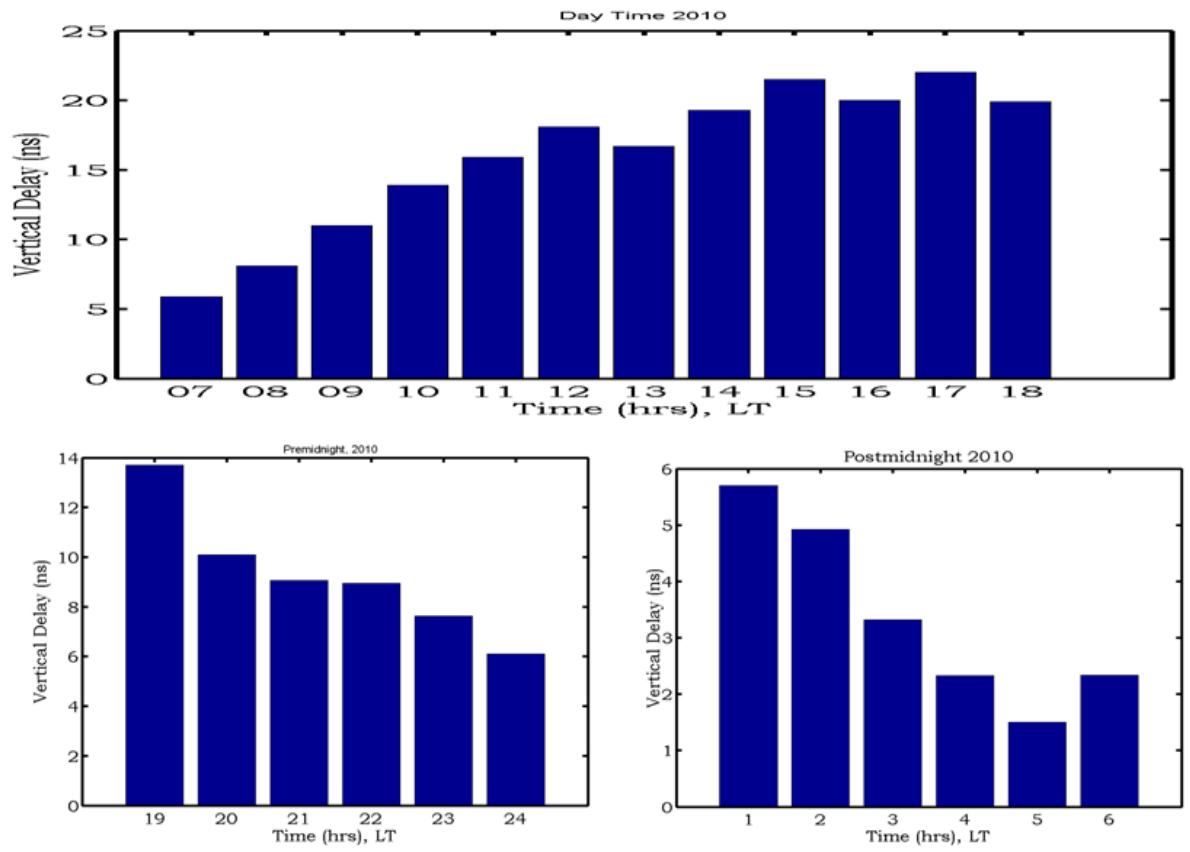

Fig. 2. Variability of the ionospheric monthly vertical time delay in September.

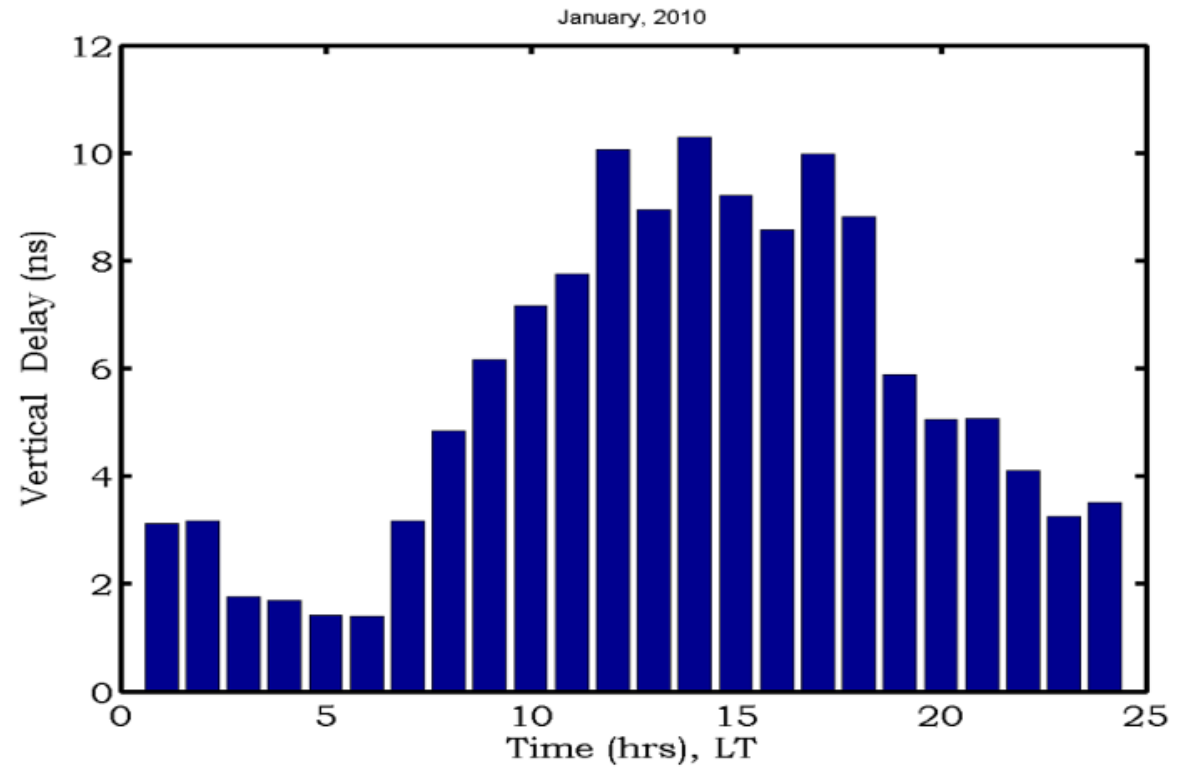

Fig. 3. Variability of the ionospheric monthly vertical time delay in January. 


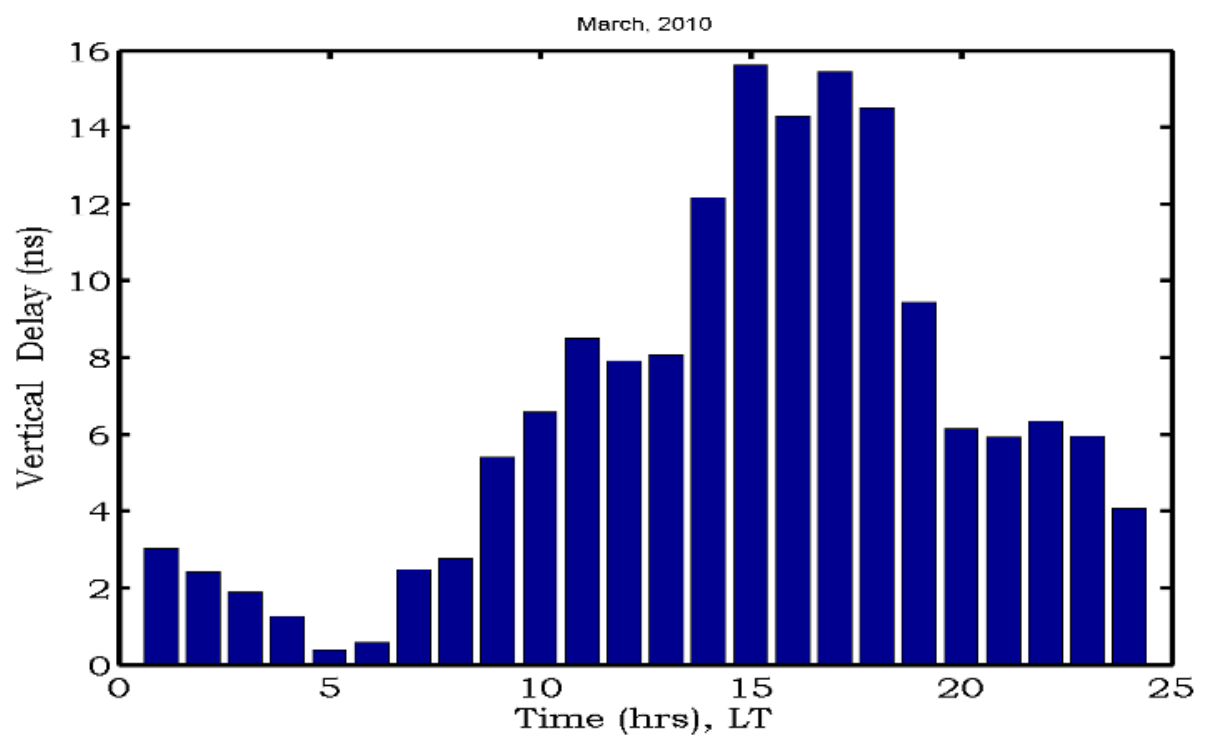

Fig. 4. Variability of the ionospheric monthly vertical time delay in March.

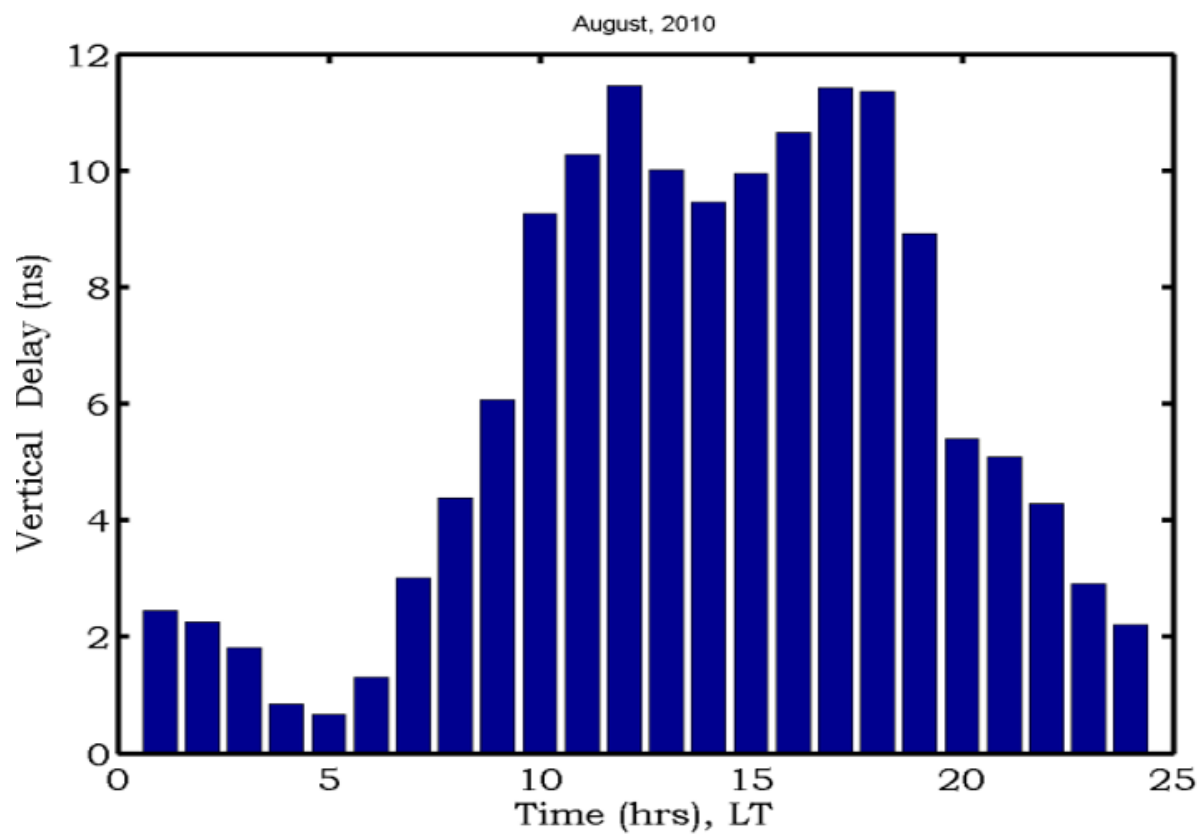

Fig. 5. Variability of the ionospheric monthly vertical time delay in August. 


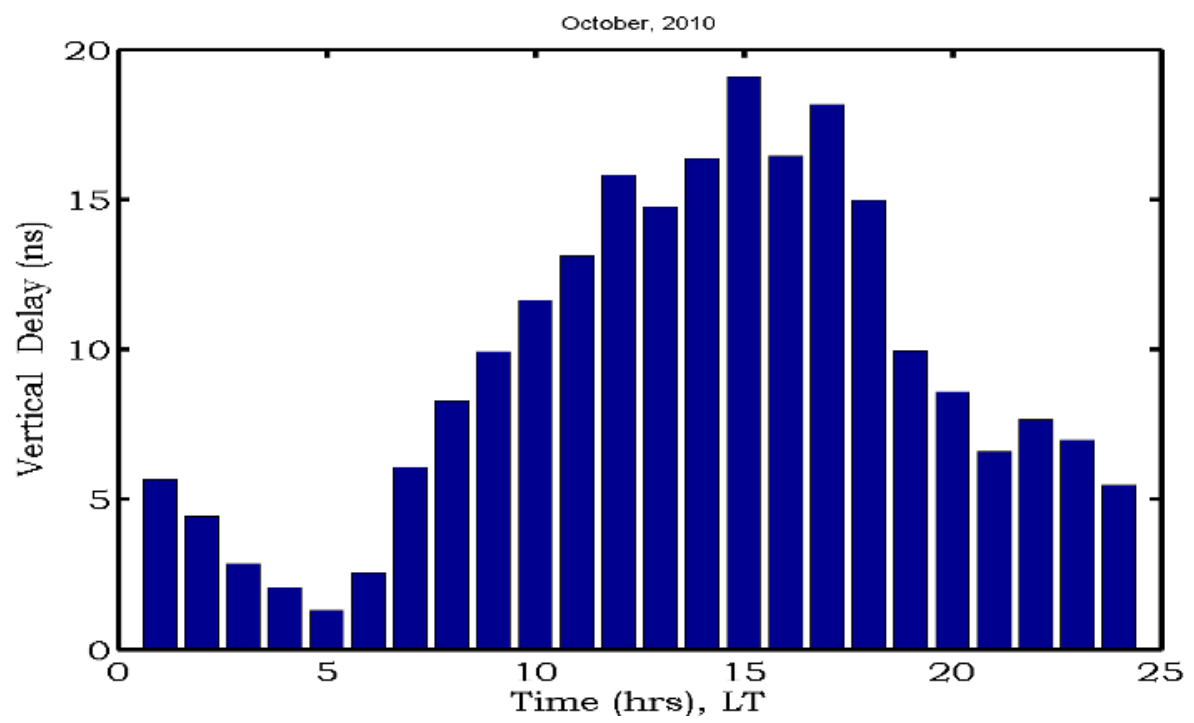

Fig. 6. Variability of the ionospheric monthly vertical time delay in October.

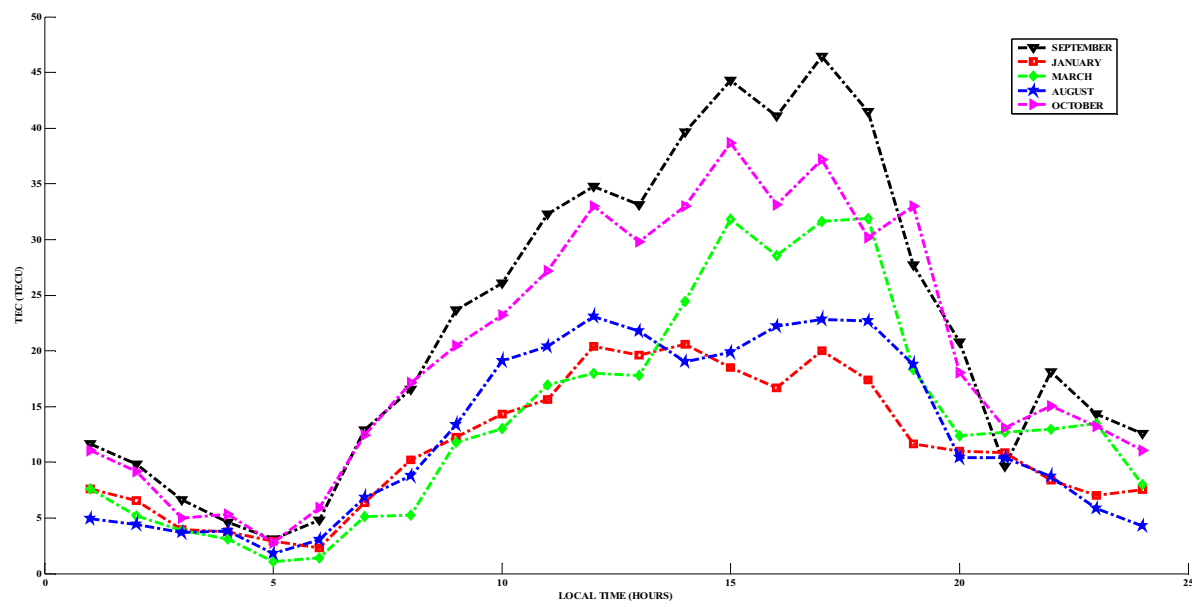

Fig. 7. Monthly mean hourly values of TEC over Akure.

Generally, all months under investigation shown in Figs. 2-6, exhibit highest, higher and low $\mathrm{M} V \Delta t$ magnitudes during daytime, pre-, and postmidnight periods, respectively. As can be observed from Fig. 2 (September), during daytime, pre-, and post-midnight hours, $\mathrm{M} V \Delta t$ magnitudes range from $\sim 6$ to $\sim 21 \mathrm{~ns}(\sim 1.80$ to $\sim 6.30 \mathrm{~m}), \sim 6$ to $\sim 14 \mathrm{~ns}(\sim 1.80$ to $\sim 4.20 \mathrm{~m})$, and $\sim 1$ to $\sim 6 \mathrm{~ns}(\sim 0.3$ to $\sim 1.80 \mathrm{~m})$, in that order. Although, in other months: January, 
March, August, and October, similar variability patterns were observed; compare with September. However, they have varying $\mathrm{M} V \Delta t$ magnitudes during daytime, pre-, and post-midnight periods. Apart from the highest $\mathrm{M} V \Delta t$ magnitudes observed in September, January has lowest magnitudes of $\mathrm{M} V \Delta t$, in the range of $\sim 4$ to $\sim 10 \mathrm{~ns}(\sim 1.20$ to $\sim 3.0 \mathrm{~m})$ on all hours. In January, during daytime hours, the $\mathrm{M} V \Delta t$ magnitude is the highest, with a value of $\sim 10 \mathrm{~ns}(\sim 3.0 \mathrm{~m})$ around 14:00 LT; during pre-midnight hours, the highest value is $\sim 6 \mathrm{~ns}(\sim 1.80 \mathrm{~m})$ around 19:00 LT; and during post-midnight hours, between 01:00 and 02:00 LT, the highest value is $\sim 4 \mathrm{~ns}(\sim 1.20 \mathrm{~m})$. Interestingly, during the period between 17:00 and 18:00 LT, MV $V t$ variability was observed to increase slightly across all months.

These observations are similar to the works of Skinner (1966), Olatunji (1967), Bolaji et al. $(2012,2013)$ at Zaria, Ibadan, and Ilorin, respectively. They reported highest magnitudes of total electron content (TEC) over Nigeria during daytime hours using a Global Positioning System (GPS) facility. They reported that the range in maximums during daytime TEC in all months is greater that the range in minimums observed at nighttimes: preand post-midnight. They attributed this greater magnitude of TEC during daytime to greater solar ionization in-place, when the ionization loss rate is smaller. In addition to the similar highest magnitudes of TEC and $\mathrm{M} V \Delta t$ observed during daytime hours, they also have similar variability patterns. For example, the variability patterns of TEC (Fig. 7) and MV $t$ (Figs. 2-6) are characterized with low pre-sunrise magnitudes, high daytime magnitudes, and moderate post sunset magnitudes. Also, during daytime hours, Skinner (1966), Olatunji (1967), and Bolaji et al. $(2012,2013)$ reported that the variability of TEC magnitudes was the highest in all months over all hours during daytime period. From this work, similar highest magnitudes of $\mathrm{M} V \Delta t$ were as well observed over all hours in all months during daytime hours. Although TEC is measured in total electron content units (TECU) but MV $V t$ is measured in nano seconds.

The slight increments observed in MV $\Delta t$ around 17:00 and 18:00 LT in all months were similar to the report of Koster (1972), Tyagi et al. (1982), Janve et al. (1979), Dabas et al. (2003), and Bolaji et al. (2012) on TEC magnitude variability. They suggested that such a scenario of slight increment of TEC magnitude during 17:00 and 18:00 LT could be due to evening renewal of fountain effect, which results from strong upward reversal of F-region EXB drift at equatorial latitudes and development of large prereversal enhancement (PRE) velocity.

Higher and lowest magnitude of $\mathrm{M} V \Delta t$ observed during pre- and postmidnight hours, respectively, could be due to higher ionization loss rate. Skinner (1966), Olatunji (1967), and Bolaji et al. (2012, 2013) observed similar results from their TEC magnitudes during pre- and post-midnight hours. 


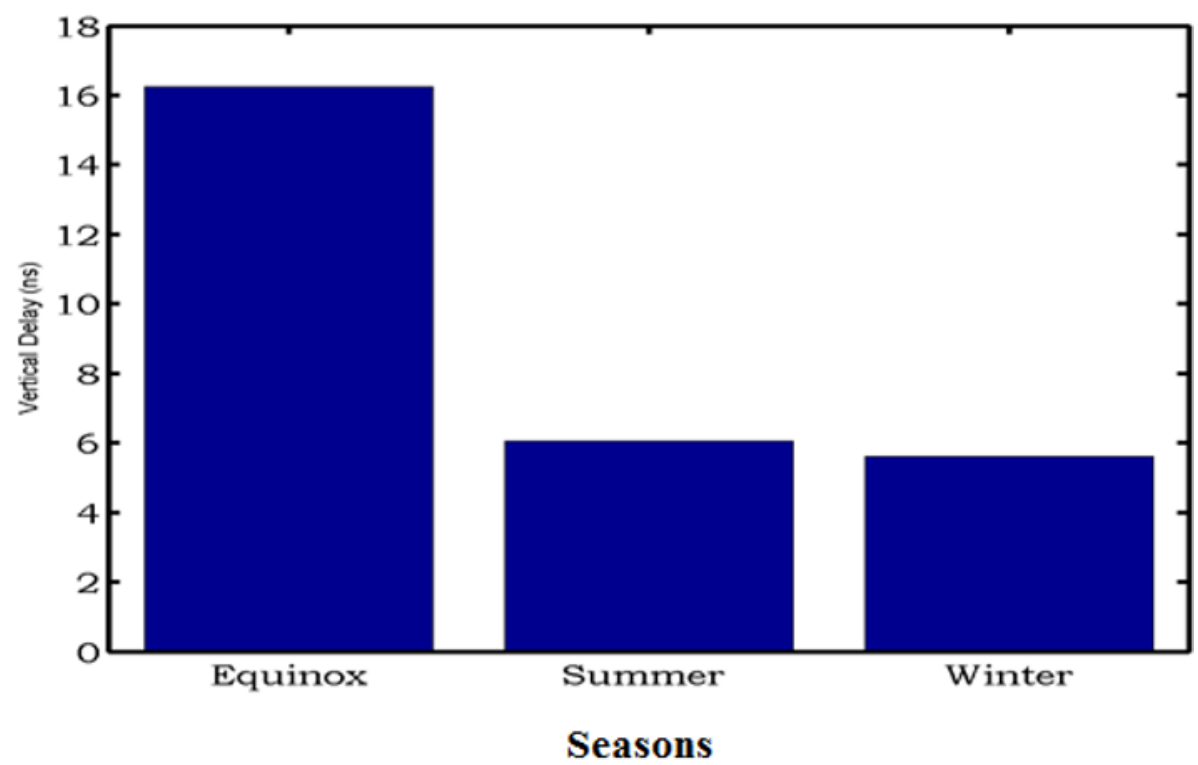

Fig. 8. Seasonal variation of ionospheric time delay in 2010.

The TEC magnitudes they observed are minimal ( 4-5 TECU) during postmidnight hours and appreciable (of 11-12 TECU) during pre-midnight hours. Apart from these similarities, the comparison is also evident from Eq. 8, which shows convincingly that there is a strong proportionality between ionospheric TEC and $V \Delta t$ parameters. Hence, this work reveals that the higher the magnitude of $V \Delta t$, the higher the magnitude of TEC, hence, otherwise. This indicates that the higher the level of electron contents in the ionosphere, the longer the delay in time of the GPS signal in the ionosphere before getting to the ground based receiver. Therefore, mechanisms responsible for TEC variability could as well directly influence the $\mathrm{M} V \Delta t$ variability.

The estimated average value of $V \Delta t$ in the year 2010 is $10 \mathrm{~ns}$ using the method of Bhattacharya et al. (2009). Our analysis has shown that for a factor of $1 \mathrm{~ns}$ for $30 \mathrm{~cm}$ in range (altitude), the average range error for the year 2010 is $\pm 3 \mathrm{~m}$ in Akure. Then, on assumption that the GPS receiver is at an altitude of $100 \mathrm{~m}$ a.s.1., there could be an equivalent reading of $97 \mathrm{~m}$ altitude recorded on the GPS. From our results, maximum daytime and postmidnight $V \Delta t$ was $\sim 21$ and $\sim 6 \mathrm{ns,} \mathrm{in} \mathrm{that} \mathrm{order,} \mathrm{which} \mathrm{is} \mathrm{equivalent} \mathrm{to} 6.30$ and $1.80 \mathrm{~m}$, respectively. These indicate that the convenient period when GPS could be best utilized is during post-midnight hours. Bhattacharya et al. (2009) investigated ionospheric time delay during disturbed period in the year 2005, a year of minimum solar activity. They observed that during the 
main phase of the storm on 24 August 2005, the ionospheric time delay has a maximum value of $36.87 \mathrm{~ns}(11.061 \mathrm{~m})$. This value is higher than the maximum value of $48 \mathrm{~ns}(14.4 \mathrm{~m})$ observed in April when there was no storm. This value (48 ns) was as well higher than the maximum value (21 ns) we got from our investigation. Hence, we suggest that these differences could result from the sunspot number. Although the years 2005 and 2010 are both classified as low solar activity years. However, the SIDC archives show that the years 2005 and 2010 had annual sunspot number of 29.8 and 16.5, respectively. They also observed reduction from 12 to 7 in the observable satellites. The consequence could lead to data loss, cycle slip, and satellite geometry disturbances. Therefore, during a severe storm period with higher disturbed storm-time (Dst) magnitudes that could spread from hours to days, $V \Delta t$ could be between 60 and $100 \mathrm{~ns}$. This will greatly affect the positioning estimate of the GPS, for example, if a severe storm period occurs when an aircraft is landing in Lagos airport, which is about $150 \mathrm{~m}$ a.s.l. The GPS facility monitoring the landing could have an equivalent altitude reading of $250 \mathrm{~m}$. This could lead to airplane crash, if the pilot had no experience about stormy period. Also, military operations could be greatly affected, as the position error of about $1 \mathrm{~m}$ could alter the exact landing position of a missile on a target. This could lead to damaging of wrong facilities, infrastructures, and loss of lives.

\subsection{Seasonal variability of vertical time delay $(S S V \Delta t)$}

The seasonal variations of $V \Delta t(S S V \Delta t)$ for all the months under investigation are shown with bar charts and their magnitudes coloured blue in Fig. 6 . The highest $S S V \Delta t$ was observed during equinoctial season with a value of $\sim 16 \mathrm{~ns}$. The value of $S S V \Delta t$ was higher and lowest in summer ( $\sim 6 \mathrm{~ns})$ and winter ( $\sim 5 \mathrm{~ns})$ seasons, respectively. This clearly shows a semi-annual variation in $S S V \Delta t$, highest value of $S S V \Delta t$ in equinoctial months (March, September, October), moderate values in summer month (August), and lower values in winter month (December). In general, our previous results and observations have shown that there is a proportionality in quantities between TEC and $V \Delta t$ variability (Eq. 8). Therefore, the mechanism responsible for seasonal TEC could be responsible for $S S V \Delta t$ as well.

Danilov and Lastovicka (2001) and Bhattacharya et al. (2009) have reported highest ionization value in the ionosphere regarding equinoctial season during disturbed periods. They attributed these highest values during equinoctial months to the down welling of thermospheric gas without any significant change in atom to molecule ratio, which is mainly caused by storm-induced thermospheric winds. But this study was carried out when there was no disturbed activity; hence, storm-induced thermospheric wind 
might not be responsible for the highest $S S V \Delta t$ observed in this study during equinoctial months. Since the ionosphere is made up of neutral oxygen $(\mathrm{O})$ and nitrogen gases $N_{2}$ (Risbeth and Setty 1961), the seasonal variability in $S S V \Delta t$ could be attributed to the changes in the ratio of atomic oxygen and molecular nitrogen gases concentration in the F2 layer, and possibly affects the F2 layer topside. Risbeth and Setty (1961) have also reported that irrespective of the solar extreme ultraviolet (SEU) radiation magnitude, the absorptions of SEU radiation in the ionosphere are always the highest during equinoctial months. This could be responsible for highest $S S V \Delta t$ during equinoctial months.

Previous works by Bhuyan and Borah (2007) regarding seasonal variability of TEC have also shown higher TEC magnitude during winter season as compared to summer season. They reported that this higher magnitude during winter season is due to weaker recombination of particles in the atmosphere. However, our results have shown higher magnitudes of $S S V \Delta t$ during summer season as compared to winter season. In this scenario, the recombination of particles in the atmosphere could be slightly higher. This could lead to lower $S S V \Delta t$ in winter as compared to summer season.

The semi-annual variations of TEC have been reported by Olatunji (1967), Bailey et al. (2000), Wu et al. (2004), Rama Rao et al. (2006), Liu et al. (2008), Lee et al. (2010), and Bolaji et al. (2012, 2013). They reported that semi-annual variation results from interaction between the magnetic field geometry and noon solar zenith angle, which is a significant factor in the increment and production of ionization during equinoctial months. Risbeth and Setty (1961) and Bhuyan and Borah (2007) have also shown that the semi-annual effect at the low latitude is caused by semi-annual changes in the neutral composition of the atmosphere.

\section{CONCLUSIONS}

The higher values of $V \Delta t$ irrespective of months and seasons are equivalent to higher values of TEC in a similar region. Therefore, our results have shown significant equivalency in terms of magnitude signature between $V \Delta t$ and TEC. Hence, mechanisms responsible for $V \Delta t$ could be responsible for TEC. We therefore conclude that telecommunications, geodetic and navigation systems will experience lesser error at night over Nigeria due to minimal $V \Delta t$ values observed. Hence, users of GPS applications like the military, security agencies, and aviation sector could take advantage of this period for operations and better services.

Acknowledgements. Authors acknowledged the kind gesture of the Air Force Research Laboratory (AFRL), USA, through its principal investigator (PI) Dr. Keith Grooves, that donated a Unit of Global Positioning Sys- 
tem (GPS) to our Space Physics Laboratory, Department of Physics, Federal University of Technology, Akure, Nigeria. This GPS Unit was donated under the Scintillation Network Decision Aid (SCINDA) project of AFRL during the campaign of the International Heliophysical Year (IHY) in 2005 under the auspices of the United Nations (UN).

\section{References}

Bailey, G.J., Y.Z. Su, and K.I. Oyama (2000), Yearly variations in the low-latitude topside ionosphere, Ann. Geophys. 18, 7, 789-798, DOI: 10.1007/s00585000-0789-0.

Battacharya, S., P.K. Purohit, and A.K. Gwal (2009), Ionospheric time delay variations in the equatorial anomaly region during low solar activity using GPS, Indian J. Radio Space Phys. 38, 5, 266-274.

Bhuyan, P.K., and R.R. Borah (2007), TEC derived from GPS network in India and comparison with the IRI, Adv. Space Res. 39, 5, 830-840, DOI: 10.1016/ j.asr.2006.12.042.

Bolaji, O.S. (2012), Variability of total electron content and magnetic field intensity at Ilorin, Ph.D. Thesis, University of Ilorin, Nigeria.

Bolaji, O.S., J.O. Adeniyi, S.M. Radicella, and P.H. Doherty (2012), Variability of total electron content over an equatorial West African station during low solar activity, Radio Sci. 47, 1, RS1001, DOI: 10.1029/2011RS004812.

Bolaji, O.S., J.O. Adeniyi, I.A. Adimula, S.M. Radicella, and P.H. Doherty (2013), Total electron content and magnetic field intensity over Ilorin, Nigeria, J. Atmos. Sol.-Terr. Phys. 98, 1-11, DOI: 10.1016/j.jastp.2013.02.011.

Chen, F.F. (1984), Introduction to Plasma Physics and Controlled Fusion. Volume 1: Plasma Physics, 2nd ed., Plenum Press, New York, 421 pp.

Dabas, R.S., L. Singh, D.R. Lakshmi, P. Subramanyam, P. Chopra, and S.C. Garg (2003), Evolution and dynamics of equatorial plasma bubbles: Relationships to ExB drift, postsunset total electron content enhancements, and equatorial electrojet strength, Radio Sci. 38, 4, 1075, DOI: 10.1029/ 2001RS002586.

Danilov, A.D., and J. Lastovicka (2001), Effects of geomagnetic storms on the ionosphere and atmosphere, Int. J. Geomagn. Aeronom. 2, 3, 209-224.

Davies, K. (1990), Ionospheric Radio, IEE electromagnetic waves series, Vol. 31, Peter Peregrinus Ltd., London, $580 \mathrm{pp}$.

Eleman, F. (1973), The geomagnetic field. In: A. Egeland,, Ø. Holter, and A. Omholt (eds.), Cosmical Geophysics, Chp. 3, Scandinavian University Books, Oslo, 45-62. 
Ginzburg, V.L. (1970), The Propagation of Electromagnetic Waves in Plasmas, 2nd ed., Pergamon Press, Oxford.

Hall, M.P.M., L.W. Barclay, and M.T. Hewitt (eds.) (1996), Propagation of Radiowaves, IEE Press, London, $446 \mathrm{pp}$.

Hofmann-Wellenhof, B., H. Lichtenegger, and J. Collins (2012), Global Positioning System: Theory and Practice, 5th rev. ed., Springer Verlag, Wien, 382 pp., DOI: 10.1007/978-3-7091-6199-9.

ISR (2009), Global Positioning System total electron content analysis application user's manual, Institute for Scientific Research, Boston College, Chestnut Hill, USA.

Janve, A.V., R.K. Rai, M.R. Deshpande, R.G. Rastogi, A.R. Jain, M. Singh, and H.S. Gurm (1979), On the nighttime enhancements in ionospheric total content at low latitudes, Ann. Geophys. 35, 159-165.

Koster, J.R. (1972), Equatorial scintillation, Planet. Space Sci. 20, 12, 1999-2014, DOI: 10.1016/0032-0633(72)90056-6.

Langley, R., M. Fedrizzi, E. Paula, M. Santos, and A. Komjathy (2002), Mapping the low latitude ionosphere with GPS, GPS World 13, 2, 41-46.

Lee, C.C., Y.J. Chuo, and F.D. Chu (2010), Climatology of total electron content near the dip equator under geomagnetic quiet-conditions, J. Atmos. Sol.Terr. Phys. 72, 2-3, 207-212, DOI: 10.1016/j.jastp.2009.11.011.

Liu, C., M.L. Zhang, W. Wan, L. Liu, and B. Ning (2008), Modeling M(3000)F2 based on empirical orthogonal function analysis method, Radio Sci. 43, 1, RS1003, DOI: 10.1029/2007RS003694.

Mannucci, A.J., B.D. Wilson, and C.D. Edwards (1993), A new method for monitoring the Earth's ionospheric total electron content using the GPS global network. In: Proc. 6th Int. Tech. Meet. Satellite Division of the Institute of Navigation, 22-24 September 1993, Salt Lake City, USA, ION GPS-93, 1323-1332.

McNamara, L.F. (1991), The Ionosphere: Communications, Surveillance, and Direction Finding, Orbit - a foundation series, Krieger Publ. Co., Malibar.

Odijk, D. (2002), Fast Precise GPS Positioning in the Presence of Ionospheric Delay, Publications on geodesy, Vol. 52, Netherlands Geodetic Commission, Delft, $242 \mathrm{pp}$.

Olatunji, E.O. (1967), The total columnar electron content of the equatorial ionosphere, J. Atmos. Terr. Phys. 29, 3, 277-285, DOI: 10.1016/0021-9169(67) 90197-3.

Rama Rao, P.V.S., S. Gopi Krishna, K. Niranjan, and D. Prasad (2006), Temporal and spatial variations in TEC using simultaneous measurements from the Indian GPS network of receivers during low solar activity period of 20042005, Ann. Geophys. 24, 12, 3279-3292, DOI: 10.5194/angeo-24-32792006. 
Ratcliffe, J.A. (1975), The early ionosphere investigations of Appleton and his colleagues, Phil. Trans. Roy. Soc. London A 280, 1293, 3-9, DOI: 10.1098/ rsta.1975.0088.

Rishbeth, H., and C.S.G.K. Setty (1961), The F-layer at sunrise, J. Atmos. Terr. Phys. 20, 4, 263-276, DOI: 10.1016/0021-9169(61)90205-7.

SIDC (2013), Solar Influences Data Analysis Center, archive at http://sidc.oma.be/ sunspot-data (assesses 14 July 2013).

Skinner, N.J. (1966), Measurements of total electron content near the magnetic equator, Planet. Space Sci. 14, 11, 1123-1129, DOI: 10.1016/0032-0633 (66)90026-2.

Tyagi, T.R., K.C. Yeh, A. Tauriainen, and H. Soicher (1982), The electron content and its variations at Natal, Brazil, J. Geophys. Res. 87, A4, 2525-2532, DOI: $10.1029 / J A 087 i A 04 p 02525$.

Wu, C.C., C.D. Fry, J.Y. Liu, K. Liou, and C.L. Tseng (2004), Annual TEC variation in the equatorial anomaly region during the solar minimum: September 1996 - August 1997, J. Atmos. Sol.-Terr. Phys. 66, 3-4, 199-207, DOI: 10.1016/j.jastp.2003.09.017. 